\title{
Uptake and tolerability of repeated mucosal specimen collection in two Phase 1 AIDS preventive vaccine trials in Kenya
}

\author{
G Mutua ${ }^{1}$, G Omosa-Manyonyi ${ }^{1}$, H Park ${ }^{2 *}$, P Bergin ${ }^{3}$, D Laufer ${ }^{2}$, PN Amornkul ${ }^{2}$, J Lehrman², P Fast ${ }^{2}$, J Gilmour ${ }^{3}$, \\ O Anzala ${ }^{1}$, B Farah ${ }^{1}$
}

From AIDS Vaccine 2012

Boston, MA, USA. 9-12 September 2012

\section{Background}

Mucosal specimens are useful to evaluate local immune responses in AIDS preventive vaccine trials, but the acceptability and tolerability of mucosal sampling in Africa remains unknown.

\section{Methods}

The Kenya AIDS Vaccine Initiative (KAVI) initiated two AIDS preventive vaccine trials in Nairobi in 2011. After informed consent for a mucosal substudy, participants were asked to provide any of several types of mucosal secretions: saliva, oral fluids, semen, cervico-vaginal and rectal. Specimens were collected at baseline, one month after final vaccination, and at the next scheduled trial visit. A tolerability questionnaire was administered at the final visit.

\section{Results}

Of 80 trial participants, $65(81.3 \%)$ consented to the mucosal sub-study and provided at least one specimen, 7/65(10.8\%) gave all specimens at least once and 2/65 (3.1\%) gave all possible specimens at all visits. Saliva and oral fluids were given at all time-points by $62 / 65(95.4 \%)$ participants. Of 48 men, 21(43.8\%) provided semen at baseline, 18/21 completed all 3 time-points. Of 17 women, $15(88.2 \%)$ gave vaginal sponge and SoftCup specimens at least once; 8/15(53.3\%) gave both at all eligible time-points. Rectal sampling was the least acceptable method: $13 / 65(20 \%)$ participants agreed at baseline [4/17 women (23.5\%), 9/48 men (18.8\%)]. Of these, 4 men and 2 women gave samples at all time-points. The most common reason for accepting mucosal sampling was a desire to contribute to HIV research and for refusal, embarrassment/emotional discomfort.

\section{Conclusion}

Repeated saliva, oral fluid, semen and cervico-vaginal mucosal sampling in AIDS vaccine preventive trials in Kenya is feasible; this study however re-affirms the challenge of repeated rectal mucosal sampling in low-risk participants, noted in an earlier observational study at KAVI (AIDS Vaccine 2010 P10.07). Possible explanations include cultural and religious reasons contributing to embarrassment and emotional discomfort in low-risk participants. Including more qualitative research in vaccine trials with mucosal sampling could help elucidate these findings.

\section{Author details}

${ }^{1}$ Kenya AIDS Vaccine Initiative, Nairobi, Kenya. ${ }^{2}$ International AIDS Vaccine Initiative, New York, NY, USA. ${ }^{3}$ International AIDS Vaccine Initiative Human Immunology Laboratory, London, UK.

Published: 13 September 2012

doi:10.1186/1742-4690-9-S2-P122

Cite this article as: Mutua et al:: Uptake and tolerability of repeated mucosal specimen collection in two Phase 1 AIDS preventive vaccine trials in Kenya. Retrovirology 2012 9(Suppl 2):P122.

${ }^{2}$ International AIDS Vaccine Initiative, New York, NY, USA

Full list of author information is available at the end of the article

(C) 2012 Mutua et al; licensee BioMed Central Ltd. This is an Open Access article distributed under the terms of the Creative Commons 